\title{
How IoT Helps Freelancers with a Gadget
}

\author{
Farzad Paridar and Mohammad Reza Majma
}

\begin{abstract}
We can observe many examples of Internet of Things (IoT) around us and the number of these instances is increasing in line with technological advances. The commercial and business sectors are the greatest consumers and users of IoT solutions. This study aims to examine IoT connection in a wider area of people's life and career, namely, IoT connection to freelancers. There are millions of freelancers throughout the world working in different fields including Information Technology (IT), Art, Music, and other great industries; and the number of these freelancers is on the increase. A gadget connected to IoT can introduce fundamental changes in the activity trends of freelancers throughout the world. Such a gadget is proposed in this study. This gadget has been patented and can provide a suitable solution for managing daily activities of a freelancing person.
\end{abstract}

Index Terms-Component, ITO, freelancers, gadgets, 2lemetry.

\section{INTRODUCTION}

The IoT ecosystem provides users (whether individuals or sectors) with the possibility of connect to and controlling IoT-based tools. In this ecosystem, users use different remote control devices such as smart mobile phones or tablets to send commands or messages for receiving information through a network to an IoT-based device/tool. Each tool then either executes the received command itself or sends the received information for being processed or displayed in another remotely-controlled device. IoT is a concept and an example acting through pervasive presence in the environment of various objects to enable - via wired or wireless communication or exclusive addressing schemes, to interact with one another and cooperate with other objects for creating applications and services aimed at achieving common objectives. There are numerous challenges, however, in the way of developing a smart world in this regard. The purpose of IoT is to enable objects to ideally communicate - at any time and place- with other objects or persons through a path/network or another service.

For example, let us consider the smart home scenario. Since television and Xbox are often placed together in the sitting room, the relationship between them is defined as that existing between devices situated in one place. With the rapid development of various microchips, sensor devices, networks, and software in recent years, IoT has gained popularity in many parts of the world [1]. One specific example is the Industrial Logistics Company where IoT is utilized in many

Manuscript received April 9, 2017; revised June 15, 2017.

Farzad Paridar is with the Network Engineering, Islamic Azad University Electronic Branch, Tehran, Iran (e-mail: Farzad.paridar@me.com).

Mohammad Reza Majma is with the Department of Computer Engineering, Pardis Branch, Islamic Azad University, Tehran, Iran (e-mail: m_majma@pardisiau.ac.ir). aspects including tracing packages, localizing delivery, and supervising vehicles. Another scenario is the Smart Home concept which is among the hottest applications in people's daily lives. The advantages offered by IoT-based technology and home automation systems have life-transforming potentials and can be implemented to improve users' comfort and provide them with such facilities as easier device operation, energy consumption efficiency, and enhanced security when controlling their lighting, air-conditioning, and heating/cooling, home appliance, communication, entertainment, and security systems. Researchers are pushing for green IoT communication by making use of efficient activity scheduling techniques to save energy [2].

In line with the evolution of IoT, networks shall also be connected to the security, analysis, and management capabilities, and in some cases and even provide convergent operations in certain cases. Fig. 1 shows IoT as a network of networks.

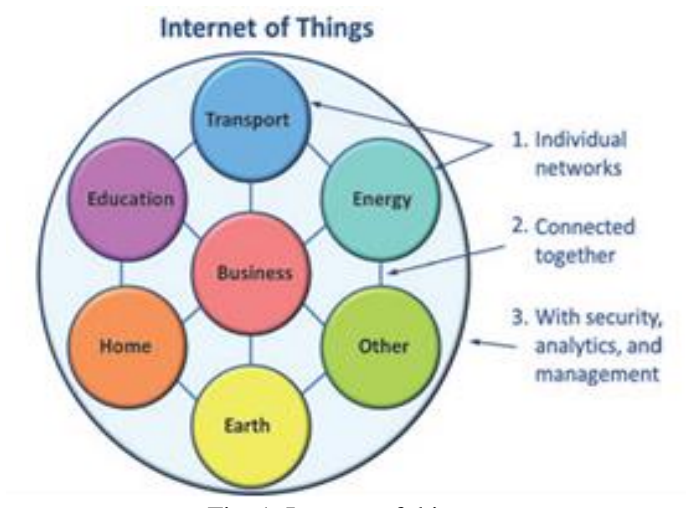

Fig. 1. Internet of things.

It is predicted that by 2020 , more than 30 billion connected mobile objects with over 200 billion alternative communications will have been provided. The key technologies include integrated sensors, image recognition, and near field communication (NFC). According to Zhou et al. there are different types of IoT sensor which are varied with different kinds of standards and technology for network communication technology [3]. IoT is a global concept and requires a common definition. Duo to the broad themes and technologies required, from highly sensitive devices to communication, data collection, and data preprocessing systems in the object sample, and ultimately, providing services, this makes the definition of IoT very hazy and poses a challenge.

\section{INNOVATION IN THE FIELD OF IOT}

Developing various technologies including nanoelectronics, communication, sensors, smart mobile phones, integrated systems, cloud networks, network 
virtualization, and software is essential for providing the capacity to connect objects anywhere and at anytime. Such developments shall also support innovation in key future IoT products, thus affecting numerous industrial sectors [1]. Some of these technologies such as integrated or cyber-physical systems forming the edges of IoT shall fill the existing gap between the cyber world and the physical world of real objects. These are also vital for activating IoT capabilities for transferring vital insight and can become a part of larger systems in the "systems of systems" world [1].

Thus, innovation in IoT era is based on three basic factors, including "increase performance, data and IO bandwidth per watt" [4].

\section{APPLIED IOT OUTLOOK}

A module for interacting with the IoT-based local devices (integrated, for example, in mobile phones or placed in the vicinity of the user to provide short-range wireless communication). This module shall be responsible for obtaining observational data and sending them to remote services for due analysis and permanent storage.

A module for conducting local analysis and processing the observational data obtained from IoT devices.

A module for direct interaction with remote IoT devices through the Internet, or, in a more likely scenario, via a proxy. This module shall be responsible for obtaining the observational data and sending them to remote services for due analysis and permanent storage.

A module for processing and analyzing special data in applications. This module would be running on an application server for providing service to all customers. This module is obtained from the requests placed by mobile phone and web customers as well as the IoT-related observations such as inputs, execution of data processing algorithms, and generation of output with due consideration of the knowledge that would be subsequently presented to the users.

A module for integrating the IoT-based information generated during a company's commercial processes. The significance of this module would increase with increasing use by companies of IoT-based data as an important part of performing daily commerce or defining commercial strategies.

Web or mobile user interface: virtual display of the measuring conducted in a particular field (e.g., a map) and interaction with the user on such subjects as defining the user's doubts and questions

Highlighting the fact that a vital factor in the success of IoT would be to convert vertical-oriented and closed systems into open systems based on open APIs and standard protocols at various levels.

Steegen argues that "a reduction of storage power is needed to enable the low power requirement for devices in the Internet of Things" [4].

\section{FREelancers' STATUS IN THE World}

Freelancers are individuals specialized in one or more fields who work freely with others on specific projects.
Statistics conducted by the end of 2015, there are currently 53 million freelancers working on various projects, comprising $34 \%$ of the workforce in the United States. The remarkable point is that $69 \%$ of these freelancers believe technology has made finding and managing their freelancing work simpler. Freelancers' economic contribution to their country is estimated at 715 billion dollars. Due consideration of theses statistics as well as the technological effects associated with this extensive economic area shows that IoT can play a significant role in improving the performance of freelancers as a major social group.

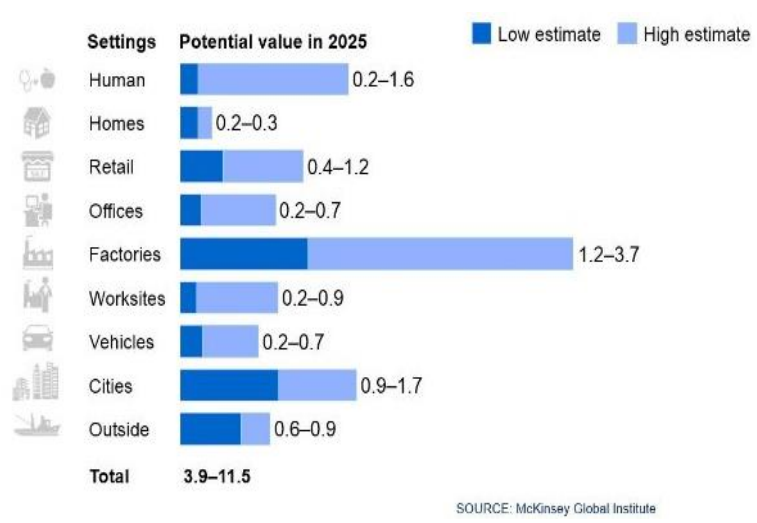

Diagram. 1. IoT can enable \$3.9-11.1 trillion in value in 2025 .

The diagram above shows the huge financial benefits that can be accrued from IoT. By 2025 an estimated value of $\$ 3.9$ to $\$ 11.1$ trillion is expected to be generated.

Also, the projected revenue growth of IoT globally is displayed in the diagram below.

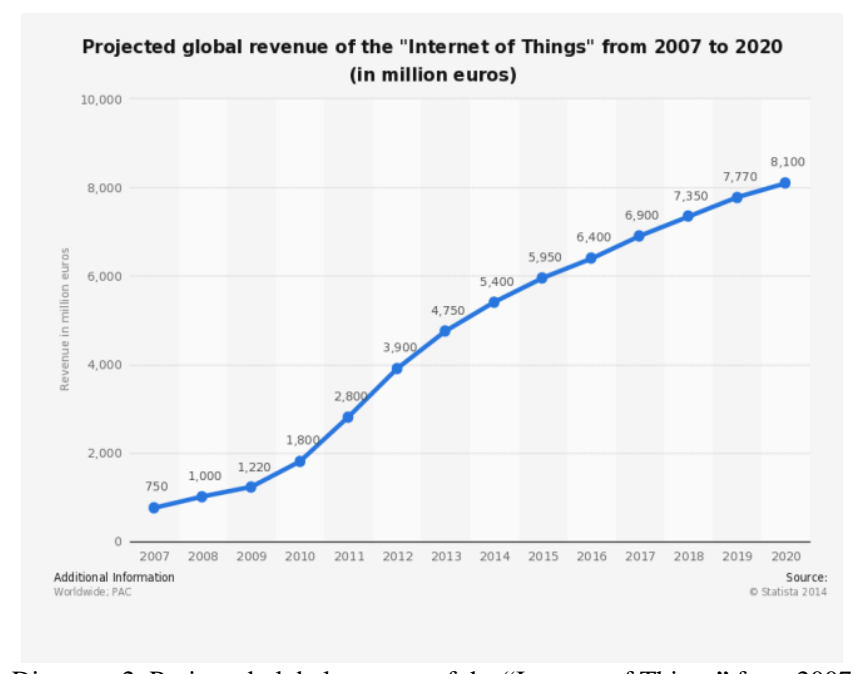

Diagram. 2. Projected global revenue of the "Internet of Things" from 2007 to 2020 (in million euros).

The diagram, thus, shows that by 2020 about 8,100 million euros in revenue would be generated; a high increase from the $\$ 750$ million gotten in 2007 .

\section{INTEGRATING INTERNET INTO THE WORLD OF FREELANCERS}

This study aims to propose a solution for making practical use of IoT to improve freelancers' daily performance and activity management. "The process of merchandising is the 
coupling process of IoT technology innovation and business modes innovation" [5]. Electronic gadgets, robots, and wearable devices are next generation's most important tools. In the same way wearable devices like smart watches are replacing smart phones, robots and gadgets can be combined with IoT to play especially fundamental roles in this field. The proposed gadget can be connected to the Internet and, upon being allocated an IP Version 6, synchronized with its mobile application. Most Internet standards are too complex for the constrained devices in the IoT. Thus data silos are created as because the devices are designed to run proprietary protocols [1]. The user can carry the gadget around or use it in her office. The gadget displays the freelancer's daily management plan on its display. Any messages that might be sent to the freelancer by the Employer can also be displayed. This gadget is capable of broadcasting audio messages from users or the Employer, and can display the received messages or the freelancer's daily program. Upon being synchronized with the application coded under Android or i-Phone, the gadget can ideally display all the required information for the user in a precisely scheduled format [1]. Other information including financial transaction management and financial inputs/outputs can also be displayed. The eyes of the gadget can change color to show your active/passive functions, or even indicate the status of receiving a project. Taking advantage of artificial intelligence, you can automatically manage your projects, your responses to projects, and the bids you send to the relevant parties. All these capabilities are possible thanks to the IoT. In addition, argue that services should accomplish the IoT technological infrastructure to enable more human activities have smart environment for the applications domains [6].

The architecture of the proposed gadget can be implemented via 2elementary. The capability called 2elementary provides IoT customers with fast integrated products and inventions along with their inherited hardware as well as software, thus presenting performances on a scale far superior to the solutions presented in current markets. 2elemetry's ThingFabric ${ }^{\mathrm{TM}}$ platform is designed for creating superior intelligence, performance, and flexibility. The main core of this platform architecture is a broker which coordinates all communication among the devices and components integrated in the platform. This broker was designed using multi-function nodes in a peer-to-peer (P2P) architecture.

As compared with other protocols, 2 elementary supports many current rules such as open publishing, SMS, Email, $\operatorname{HTTP}(\mathrm{S})$ WebHooks , and the repay load format, and is capable of adapting any protocol to the user's conditions, from low payloads to highly complicated and dedicated payloads. This allows the user to experience the fast pace of innovation without being worried about basic costs, complications, delays, etc.

The 2elementary solutions are able to adjust and administer at any scale anything including IoT implementations, and can administer the great volumes of large interactions as well as device networks. The 2elementary platform can automatically and indefinitely store data, thus allowing the user to access any data at any time. In addition, all data can be stored in three different physical sites so as to ensure that there is no single point of failure (SPOF) in the system. This would also increase the error tolerance. All the capabilities are essential in an IoT-based gadget, particularly one operating at a large scale. This platform provides the possibility of adding new devices and sensors to the gadget and sending data without worrying about platform configuration. The data thus sent can be immediately accessed by the local commercial application. This would reduce costs and eliminate the complications associated with operating at a large scale. In addition, since we can work with any legacy system, there would be no need for upgrading the hardware. The 2elementary technology is capable of communication without physical contact with or even seeing a device, thus eliminating the time consuming initialization process. The proposed platform is not hardware-based, but based on software and the cloud technology.

Many competitors are attempting to overcome the communication challenges posed by IoT through hardware. In 2elementray, however, these problems are solved via software and the cloud power. "Applications in IoT and crowd sensing generate also a huge amount of information worth to be processed" [8]. The proposed dispenses with developing new SDKs for the gadget or introducing modifications in the present infrastructures to adapt them to the new protocol. Removing the major obstacles in term of IoT adaptation, this method allows the user to work fast and concentrate on her main job.

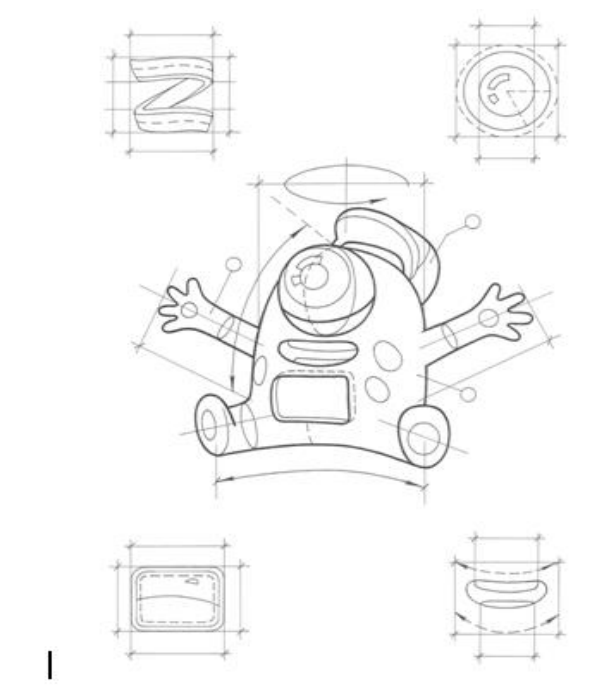

Fig. 2. Picture of required gadget prototype for freelancers

The 2elementaryplatform significantly reduces the complexity and ramp-up time associated with other IoT solutions. In addition, this unique platform enables your IoT system to adopt new capabilities while offering it high speed and incredible scale. Major priorities of IoT include self-awareness, context-awareness, and electronic networking abilities, intelligence or smartness [7]. Commercial users can more effectively model and construct the complicated processes as well as easily integrate the system data for making decisions for building devices. Through accelerated innovation and reduced costs, technical teams can concentrate exclusively on presentation values. However, the IoT industry, even with its large industrial 
chain, still has obstacles in its development [5].

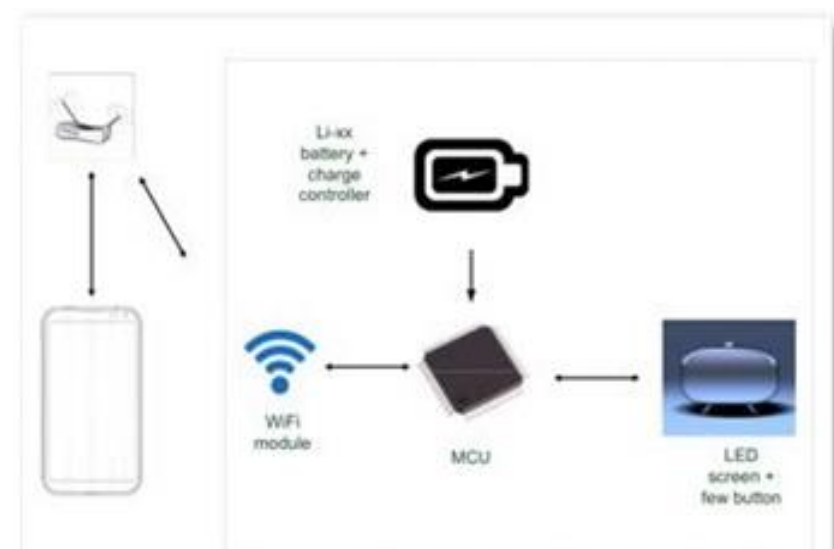

Fig. 3. Required hardware components and parts to set up at least one gadget.

According to Arauz "understanding the implications of enabling movement in IoT is key for the deployment of of actuating objects"[9]. The 2elementary platform can provide a high-performance and scalable middleware with a single development layer to as companies make decision to connect the device data to incorporate their system, including customer relationship management (CRM), marketing automation, supply chain management, and quality assurance and services. Through this, 2elementary can help a company by converting the device data into immediate practical intelligence so as to reduce costs, satisfy customers through improved service provision, and promote innovation via new products and services as well as increased income flows.

\section{THE PROPOSED HARDWARE}

Several types of smart devices are linked with IoT in contemporary times [10]. The implementation process for the proposed gadget includes the following:

- Schematic design and components

- Designing the finalized BOM

- Designing the PBCs in terms of size and quantity

- Final testing and interfacing the mobile application to the gadget as well as the IoT platform

This gadget designed for using as remote indication for smartphone events. We can distinguish the basic building blocks - the power supply, the MCU block (central processing unit with wireless transceiver) and indication (visual and acoustic) - The power supply block is using for provide stable voltage which device is needed, and also for battery charge/discharge control. Specialized IC is also monitoring the battery temperature, has current limit and voltage protection. - MCU provides $\mathrm{WiFi}$ connection and exchange data between device and smartphone, runs main program. - Indication block provides signalization or device reaction for events (from smartphone or internal, such as errors).

\section{CONCLUSION}

IoT is actually a network of Internet-based tools capable of collecting and exchanging information. IoT would virtually change the way individuals and the real world interacts with the cyber and technological worlds. The influence of IoT on different aspects of human life is increasing. In line with supplying users' demands, most IoT-based devices, rather than acting alone, establish relationships with other IoT-based devices, just as humans establish relations to cooperate with one another. Iconic financially active institutions have always attracted technology developers. IoT can be effectively used in professional and financial activities such as freelancers' in a similar way technology is used for communicating with vehicles towards ultimately making driverless intelligent cars acceptable to people.

\section{REFERENCES}

[1] P. Friess, "Internet of things: Converging technologies for smart environments and integrated ecosystems," Rivers Publishers, 2013.

[2] S. P. Shyam and C. kumar, "An energy efficient and reliable internet of things," Institute of Electrical and Electronics Engineers, October 19, 2012.

[3] M. Zhou, H. Fan, and Y. Ma, "Semantic annotation method of IOT middleware," Institute of Electrical and Electronics Engineers, June 9, 2013.

[4] A. Steegen, "Technology innovation in an IoT era," Institute of Electrical and Electronics Engineers, June 17, 2015.

[5] W. Wang and L. Liu, "Research on the coupling relations between technology innovation and business modes innovation of IOT industry based on SD," Institute of Electrical and Electronics Engineers, November 23, 2013.

[6] S. Aelita and K. Marius, "The internet of things: When reality meets expectations," International Journal of Innovation and Learning, vol. 17, no. 2, 2015.

[7] M. Forcolin, E. Fracasso, F. Tumanischvili, and P. Lupieri, "EURIDICE-IoT applied to logistics using the intelligent Cargo concept," Institute of Electrical and Electronics Engineers, January 20 , 2011.

[8] D. Sanchez, S. Sherratt, A. P. Almenarez, and M. Andres, "Enabling actor model for crowd sensing and IoT," Institute of Electrical and Electronics Engineers, June 24, 2015.

[9] J. Arauz, "The moving IoT," Institute of Electrical and Electronics Engineers, August 27, 2014.

[10] H. Sehyeon, W. Sungpil, I. Janggwan, and K. Daeyoung, "IoT-MAP: IoT mashup application platform for the flexible IoT ecosystem," Institute of Electrical and Electronics Engineers, October 26, 2015.

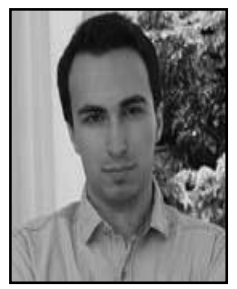

Farzad Paridar is a network computers student in Islamic Azad University Since 2015.

His current research interests include internet of things in projects, smart cities, cloud computing and data mining. He is the chairman of the board for 2 startup in IOT field.

$\mathrm{He}$ has published 5 academic papers in peer-reviewed international journals and conference.

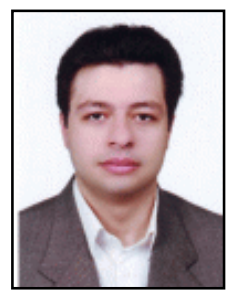

Mohammad Reza Majma received his B.Sc. in computer engineering from Islamic Azad University in 2005 and his M.Sc. from Islamic Azad University, Ghazvin branch, Iran in 2008. He received his Ph.D. degree from Islamic Azad University, Science and Research Branch, Tehran, in 2014 in computer engineering. He is the faculty member in Computer Engineering Department of Islamic Azad University, Pardis Branch, since 2009. His research interests are wireless networks (sensor networks and ad hoc), modeling evaluation of computer networks, high performance computing, grid computing, cloud computing, internet of thing and software defined networking. 